\title{
Balkanologie
}

Balkanologie Revue d'études pluridisciplinaires

Vol. IV, $n^{\circ} 2 \mid 2000$

Volume IV Numéro 2

\section{Korçë dans la Grande Guerre}

Le sud-est albanais sous administration française (1916-1918)

\section{Etienne Augris}

\section{CpenEdition}

Journals

Édition électronique

URL : http://journals.openedition.org/balkanologie/315

DOI : 10.4000/balkanologie.315

ISSN : 1965-0582

Éditeur

Association française d'études sur les Balkans (Afebalk)

Édition imprimée

Date de publication : 1 décembre 2000

ISSN : 1279-7952

Référence électronique

Etienne Augris, «Korçë dans la Grande Guerre », Balkanologie [En ligne], Vol. IV, n² 2 | 2000, mis en ligne le 20 juillet 2011, consulté le 17 décembre 2020. URL : http://journals.openedition.org/balkanologie/

315 ; DOI : https://doi.org/10.4000/balkanologie.315

Ce document a été généré automatiquement le 17 décembre 2020.

(c) Tous droits réservés 


\title{
Korçë dans la Grande Guerre
}

Le sud-est albanais sous administration française (1916-1918)

\author{
Etienne Augris
}

1 Après l'échec des Dardanelles en 1915, le corps expéditionnaire français s'installe à Salonique. Un nouveau front s'ouvre progressivement à l'est de l'Europe. Après la déroute de l'armée serbe en décembre 1915 et son évacuation par le nord de l'Albanie, le pays est occupé au nord par les Autrichiens. Les Grecs occupent le sud-est, où se trouve Korçë, depuis octobre 1914 et les Italiens le sud-ouest depuis le mois de décembre de la même année.

2 À l'automne 1916, le général Sarrail s'inquiète de ce qui constitue son flanc gauche, qui ne forme pas véritablement un front, mais où des troubles opposent les venizélistes à des bandes et à une grande partie de la population de Korçë et de ses environs.

3 C'est l'attitude de la France dans cette zone que nous allons aborder. Les militaires français sont en effet maîtres du terrain de novembre 1916 à mai 1920, date à laquelle ils abandonnent Korçë au gouvernement de Tirana auquel s'est ralliée la population. Cet article traite donc des raisons de la présence française à Korçë, puis des modalités de cette présence jusqu'à la fin de la guerre et l'attente des décisions de la Conférence de la paix au début de 1919.

\section{Les raisons de la présence française}

4 C'est avant tout pour porter secours à l'armée serbe que les Français se sont engagés en Macédoine. La partie sud-est de l'Albanie, où se trouve la ville de Korçë que les Français appellent Korytza (il s'agit du nom grec), n'est pas convoitée par les Serbes comme peut l'être le nord. Korytza est occupée par les Grecs qui y exercent une administration de fait depuis octobre 1914. Le Kaza (ancienne division administrative ottomane correspondant à une sous-préfecture) est géré successivement depuis cette date par des irréguliers (andartes), des militaires, des royalistes, puis des venizélistes. Mais, à la fin de 1916, si les Alliés ont imposé leur présence à Salonique, la Grèce n'est pas encore dans le camp de l'Entente. Elle est profondément divisée entre les partisans du roi Constantin, plutôt germanophiles à l'image du souverain, et les partisans de l'ancien 
Premier ministre Eleftherios Venizélos, favorable à l'Entente. En butte aux tracasseries des Grecs autour et dans Salonique, le général Sarrail, qui dirige l'armée alliée en Orient depuis octobre 1915, cherche à avoir les mains libres pour ne s'occuper que d'objectifs militaires qui sont, par ailleurs, très vagues. C'est dans cette optique qu'il réclame à plusieurs reprises aux gouvernements de l'Entente la proclamation de l'état de siège à Salonique qui est devenue une véritable fourmilière d'espions de toute sorte. Il n'obtient pourtant gain de cause qu'en juin 1916. L'envoi du colonel Descoins à Korytza relève de la même logique. Faire cesser aux marges de ses objectifs militaires une agitation politique. Cela lui est d'autant plus facile que Korytza se situe hors des frontières de la Grèce, ce qui rend son occupation grecque illégale. Venizélos, soucieux d'une bonne entente avec les Français, ne s'oppose d'ailleurs pas à leur arrivée et demande à son représentant à Korytza, Argyropoulo, de s'incliner.

5 Le but de l'opération est donc de mettre en sourdine les différentes influences et propagandes qui s'affrontent sur place. Le colonel Descoins est envoyé afin d'assurer le calme dans la zone. Le commandement français doit mener à bien des actions militaires autour de Monastir / Bitola (à environ 100 kilomètres de Korytza) qui a été abandonné par les troupes germano-bulgares en octobre. Descoins, ayant reçu son ordre de mission, cherche à obtenir des renseignements plus précis sur la conduite à tenir sur place ${ }^{1}$. Il reçoit le 15 novembre 1916, à Salonique, les instructions suivantes de l'Etat-major du général Sarrail : « le général ne veut à Korytza ni Grecs d'aucune sorte [royalistes ou venizélistes], ni Italiens, ni Essadistes». Tous les protagonistes cités sont pourtant les alliés des Français (à part bien sûr les royalistes grecs), mais Sarrail s'en méfie, pensant que chacun cherche à prendre des gages territoriaux pour l'aprèsguerre.

6 Les Italiens, qui sont déjà maîtres du sud-ouest de l'Albanie, cherchent à s'accaparer le sud-est. C'est pour cette raison que Sarrail refuse que la division italienne sous ses ordres à Salonique opère sur son flanc gauche et fasse ainsi la jonction avec les troupes du général Ferrero qui tiennent Vlorë. Ce serait laisser les mains libres aux Italiens pour préparer leur mainmise sur le pays. Essad Toptani, qui se cherche depuis longtemps un destin national, est le chef théorique d'un gouvernement albanais que reconnaissent en partie les Français, puisqu'ils lui adjoignent un représentant, Joseph de Fontenay puis Léon Krajewski. Ses troupes (quelques 800 hommes au départ) représentent un appoint pour les Alliés, mais Sarrail semble vouloir écarter cet homme, dont les amitiés semblent peu sûres et dont les Albanais eux-mêmes se méfient. En écartant les Grecs, qui ne sont pas, à cette date, belligérants, Sarrail empêche les royalistes d'utiliser ce qui constitue le dernier point de contact avec les Centraux et retire aux venizélistes un terrain de propagande.

7 Autrement dit, à part ces instructions, Descoins semble avoir les mains libres et pense alors établir une "administration indigène sous le contrôle français $»^{2}$. Il arrive à Korytza le 21 novembre, Argyropoulo lui transmet ses pouvoirs, non sans lui laisser des recommandations qui vont bien sûr dans le sens grec. Le colonel de Bournazel lui transmet également le commandement militaire des troupes françaises stationnées sur le territoire.

8 Sur un plan purement diplomatique, Descoins ignore tout de l'accord secret de Londres du 26 avril 1915 qui, permettant l'entrée en guerre, aux côtés de l'Entente, de l'Italie, offrait à celle-ci une partie de l'Albanie (Vlorë), laissant seulement le centre à un « petit Etat autonome neutralisé » et le reste aux Monténégrins, Serbes et Grecs. Cet accord 
envisageait en effet de céder Korytza à la Grèce ${ }^{3}$. Les objectifs de Descoins paraissent donc en contradiction avec les projets de la diplomatie française en Albanie. Mais nous sommes en période de guerre et cette diplomatie est en sommeil, du moins dans cette zone. Léon Krajewski, qui était le délégué français à la Commission internationale de contrôle mise en place à la conférence de Londres en 1913 est rentré à Paris en septembre 1915, et ne retourne dans la région qu'en 1917, pour assurer la gérance de la légation près du gouvernement albanais à Salonique, en l'occurrence celui d'Essad pacha. Le consul de Fontenay, en poste à Durrës de 1914 à 1916, va d'ailleurs se rendre à Korytza à deux reprises à la demande de Sarrail, en novembre 1916 et septembre 1917. Mais Descoins ne semble pas avoir reçu de conseils de sa part. Du moins ne le mentionne-t-il pas dans son récit de six mois de présence à Korytza.

9 Descoins obtient cependant des conseils de deux diplomates, qui ne servent que de simples intermédiaires entre lui et Sarrail. En effet, les deux diplomates en question connaissent bien la région. Il s'agit de Bargeton qui n'est qu'un fonctionnaire du Quai d'Orsay mobilisé à l'état-major de Salonique et de Berne-Lagarde, ancien consul de France à Monastir et qui, à ce titre, connaît bien la question albanaise. Si l'intervention de Berne-Lagarde est ponctuelle et ne relève pas de la diplomatie officielle, celle de Bargeton, dont la position est plus modeste, s'inscrit dans la durée puisqu'il va rester à Korytza jusqu'à la mi-janvier 1917. Nous verrons quel fut son rôle auprès du colonel Descoins.

10 Ainsi, c'est l'armée qui est maître du jeu dans les Balkans, et c'est elle qui assume l'administration des territoires où elle se trouve pour des raisons stratégiques. À savoir empêcher toute progression des forces de la Triplice dans cette zone, assurer la liaison avec les Italiens à l'ouest et couper par là même toute possibilité de liaison entre les Autrichiens et Constantin. Tout au long de la période d'occupation française, les impératifs militaires ont commandé aux décisions administratives. Ce qui fait l'originalité de cette période, c'est que ces impératifs ont alors coïncidé avec les objectifs des notables et de la population de Korytza. La France, par l'intermédiaire de son armée, adopte ainsi une politique favorable au développement du patriotisme albanais. Elle prend à rebours son attitude précédente, couronnée par le protocole secret de Londres de 1915. Ce ne sont plus les grands acteurs de la diplomatie française, comme Paul Cambon en 1913, qui décident, mais des Français qui sont tous les jours au contact des populations locales. L'action des autorités militaires est dictée par leur observation de la réalité du terrain. Pour assurer la tranquillité que lui réclament ses supérieurs, Descoins constate qu'il doit s'appuyer sur le souhait des populations et satisfaire un minimum leurs revendications d'autonomie.

11 Pour établir de façon stable le "protectorat" français, Descoins rencontre les notables, noue des contacts avec les chefs des milices (Thémistocle Germenji [Themistokli Gërmënji] dont le rôle est par la suite important et Salih Budka (Butka) que Descoins ne rencontrera jamais directement) et leur propose rapidement un cadre administratif propre à les satisfaire, voire à permettre leur enrôlement aux côtés des Français.

\section{Le protocole du 10 décembre 1916}

Dès son arrivée, Descoins, malgré sa sympathie pour les Grecs ${ }^{4}$, constate leur activisme et s'en méfie. La prise récente de Monastir semble indiquer à la population locale que les Français sont en position de force. Le front s'est stabilisé. Descoins entrevoit alors la 
possibilité de s'assurer le soutien des Albanais : «nous devions faire quelque chose qui liât à nous le peuple albanais d'une manière irrévocable et définitive $»^{5}$. Pour cela, il se réfère uniquement aux objectifs militaires qui lui ont été assignés à Salonique : «si l'intérêt politique des Albanais les poussait à mettre à profit notre présence à Korytza pour accomplir leur révolution nationaliste, notre intérêt militaire était de laisser faire cette révolution $»^{6}$.

Mais est-ce véritablement l'état d'esprit du colonel au début de décembre 1916? En écrivant ces phrases plus de dix ans après, Descoins ne tente-t-il pas de s'assurer aprèscoup une place dans l'histoire de la construction nationale albanaise ? Il semble que son état d'esprit était le même en décembre 1916, d'après le rapport qu'il envoie lui-même alors à Sarrail, le 20 décembre, où il parle «d'une population qui nous a accueillis en libérateurs $»^{7}$. Tout au plus ajoute-t-il une touche de lyrisme à cet épisode en parlant en 1929 de « révolution ». Moins enflammé, Sarrail explique au Quai d'Orsay, qui s'inquiète de ce qui constitue un précédent, qu'il n'est préoccupé que de l'aspect militaire. Il répond en termes militaires aux interrogations des diplomates : «j'ai mon flanc gauche couvert par une sorte zone tampon. Le colonel Descoins après avoir, a posteriori dans un protocole, résumé la situation créée par les habitants de Korytza, commande cette sorte de territoire confins militaires. Au point de vue militaire qui seul m'occupe, je ne puis que me féliciter de cet état de choses. $»^{8}$

Le colonel Descoins fait arrêter les principaux meneurs grecs, les envoie à Salonique et obtient, par l'intermédiaire de Berne-Lagarde, l'autorisation de Sarrail de donner satisfaction aux notables albanais. Il élabore avec le chef de bande Thémistocle Germenji le protocole qui va régler l'administration de Korytza et de sa région. Ce protocole entérine une situation de fait et cherche à organiser l'administration plus qu'à octroyer une quelconque autonomie aux populations locales. Au minimum pour assurer la tranquillité de la région, au mieux pour mettre les bandes locales de leur côté, les Français font oeuvre nouvelle. La signature du protocole a lieu en grande pompe le 10 décembre $1916^{\circ}$. Le drapeau de Skanderbeg, cravaté aux couleurs françaises, est hissé sur l'ancienne préfecture grecque. Descoins déclare que ces couleurs, de façon hautement symbolique, sont « un ombrage qui abrite mais n'écrase pas $»^{10}$.

Un Conseil d'Administration (C.A.) de 14 membres est l'organe représentant la population locale. C'est Bargeton, dont nous avons vu le rôle auprès de Sarrail, qui est le représentant de Descoins à ce C.A. (il sera remplacé en janvier 1917 par le lieutenant Siegfried). Il s'agit pour lui de contrôler davantage que de représenter. Ainsi, si le C.A. propose des noms pour les postes de fonctionnaires, c'est in fine, le commandant français qui les nomme. Le nouveau régime ne semble correspondre à aucune définition-type. Il ne s'apparente pas à une administration coloniale, les notables qui composent le C.A. ne gèrent pas non plus le territoire à leur guise. C'est donc le flou qui domine et qui permet, au départ, de satisfaire tout le monde. Les notables albanais et le nouveau préfet de police Thémistocle Germenji vont tenter de profiter de ce flou.

\section{La « République de Korçë »}

Dès le départ, Descoins s'appuie, nous l'avons vu, sur les notables, orthodoxes comme musulmans, mais cherche aussi le soutien des chefs de bande. Si Salih Budka maintient sans cesse une ligne plus ou moins hostile aux Français, Thémistocle Germenji a vite 
compris l'intérêt qu'il pouvait tirer de la situation. Il se rallie très vite aux Français et participe à la mise en place du nouveau statut du territoire. Il est nommé préfet de police de Korytza et entretient les meilleures relations personnelles avec le colonel Descoins qui semble satisfait de ses services. Le colonel propose même de le citer à l'ordre de l'armée le 10 mars $1917^{11}$. Au cours de cette période, l'administration militaire française laisse à Thémistocle Germenji et aux notables une grande marge de manoeuvre.

17 Une république de fait semble ainsi voir le jour à Korytza. Les notables du Conseil d'Administration profitent de l'absence du représentant français en mars 1917 pour transformer ce fait en droit. Cette « République Albanaise $»^{12}$ au milieu des monarchies balkaniques et européennes est une nouveauté. Elle correspond bien à l'idéal républicain et au lyrisme de Descoins, en 1917 comme en 1929. Il va en effet jusqu'à transposer à l'échelle de Korytza " l'Union Sacrée ${ }^{13}$ qui a alors cours dans les milieux politiques républicains en France. Il emploie ce terme à propos des notables qui font taire leurs divergences et leurs querelles religieuses. Le Conseil est en effet composé à parité de musulmans et d'orthodoxes. Cette vision d'une entente interreligieuse n'est pourtant pas partagée par tous. Dans un rapport de novembre 1917, le général Salle, qui a remplacé Descoins, écrit ainsi : "la coopération des chrétiens et des musulmans a donné lieu, comme il fallait s'y attendre, à de fréquentes difficultés. Elle a montré, une fois de plus qu'en Albanie, le sentiment religieux était nettement plus fort que le sentiment de patrie $»^{14}$.

On retrouve là les opinions divergentes des observateurs étrangers et notamment français sur les questions religieuse et nationale en Albanie. La religion est perçue selon le lieu, les circonstances et l'observateur comme facteur de fanatisme ou élément secondaire ayant finalement peu d'importance. La question de savoir si le sentiment religieux l'emporte à cette époque sur le sentiment national n'est pas résolue tant l'historiographie albanaise a tenté d'établir l'hypothèse inverse comme postulat de base, au risque de se tromper. Il est cependant un fait que le Conseil fonctionne convenablement et qu'il parvient en tout cas à tomber d'accord sur la proclamation d'une république. Les musulmans sont depuis longtemps acquis à la cause du nationalisme albanais, après avoir constaté l'impasse dans laquelle se trouvait l'Empire ottoman. Les orthodoxes, qui auraient pu être tentés par le rattachement à la Grèce, comme les nombreux activistes hellénophiles tentent de le prouver, ont changé d'avis lors de l'occupation grecque. De nombreux rapports militaires français font état de la méfiance et du peu d'attrait de la population de Korytza pour le rattachement à la Grèce.

Quoi qu'il en soit, le nationalisme albanais prend ses marques à Korytza, et ce parmi les orthodoxes comme parmi les musulmans. Et Descoins a choisi de ne pas l'entraver. Lorsque l'on constate les objectifs flous de l'Armée d'Orient, on perçoit de façon encore plus vive l'écart entre Descoins qui s'inscrit dans un cadre politique et dans la durée, et Sarrail qui se bat pour obtenir des renforts militaires pour un front somme toute jugé secondaire en haut lieu. Sarrail voit surtout le moyen d'assurer une tranquillité sur son flanc gauche, alors que l'arrière n'est pas sûr du fait de la quasi-guerre civile en Grèce. La conjonction de ces motivations plus ou moins militaires laisse donc libre cours à une sorte de république. Le Conseil d'Administration de Korytza s'engouffre dans la brèche et la proclame. 

tout pour déplaire aussi bien aux Italiens, qu'aux Grecs et à Essad qui se voit ainsi écarté. La diplomatie française, qui avait demandé à Sarrail des explications dès la proclamation du protocole de novembre 1916 et qui subit les pressions des venizélistes, pousse à la clôture de cette expérience. Pour le quai d'Orsay, Descoins s'est fourvoyé. Les supérieurs du colonel s'inquiètent également. Le Général Leblois, commandant de l'A.F.O., avait dès janvier envoyé à Korytza un capitaine de son deuxième bureau, l'historien de la Rome antique Jérôme Carcopino ${ }^{15}$, qui avait estimé que le colonel accordait trop de confiance aux déclarations des notables albanais dont le ralliement semblait suspect .

21 L'expérience de Korytza suscite l'intérêt en France. Robert Vaucher écrit dans L'Illustration du 7 avril 1917 un article sur la ville. M. de Maizière du Petit Parisien se rend à Korytza et rend compte de manière plutôt favorable à Descoins dans son journal le 10 mai 1917. Mais le rappel du commandant de Korytza, le 15 mai 1917, semble être le signe que l'on estime en haut lieu qu'il est allé trop loin dans l'optique de l'aprèsguerre. Certes, Briand avait soutenu, en 1915, le maintien des Alliés à Salonique comme un gage de l'influence future de la France dans la région, mais il ne s'agit pas de trop préjuger de cet avenir. Face au surgissement de la revendication albanaise, Descoins a choisi une solution qui va contre les intérêts des alliés de la France, les Grecs notamment.

De plus, se référant à l'exemple de Korytza, les Autrichiens proclament, en janvier 1917, l'autonomie de l'Albanie sous leur égide et les Italiens, en juin 1917, l'indépendance complète sous leur protection. Voulant faire taire la politique, les militaires français l'ont ainsi remise au goût du jour. Une clarification vis-à-vis de la population, des alliés italiens, des venizélistes et surtout de la diplomatie française, devient donc urgente. Les Français vont par la suite tout faire pour minimiser l'importance de la proclamation de cette "république". Ils vont être contraints de jouer sur les mots, pour réparer ce que beaucoup considèrent comme un excès d'enthousiasme. Ainsi, le consul de France à Salonique écrit-il en 1920 : «l'étendard de Skanderbeg a bel et bien été hissé à Korytza comme à Pogradec par la volonté nationale et à l'insu de nos autorités militaires $»^{16}$. C'est faire peu de cas de l'article IX du protocole du 10 décembre 1916, qui mentionne cet étendard de façon officielle. On sent dans les rapports des différents commandants du territoire qui succèdent à Descoins une volonté d'étouffer cet épisode. Le général Salle écrit le 3 juin 1918 : «je ne veux plus voir sur les pétitions et pièces venant de l'Administration, de timbres portant l'inscription "Republika", y veiller ${ }^{17}$.

Pour déterminer de façon précise le rôle des Français dans la proclamation de cette république, il faut considérer les incertitudes de cette période de guerre et la difficulté des communications. Il appartient sans doute au colonel Descoins d'avoir toléré, sinon encouragé, ce fait. Il est néanmoins juste de dire que la France, dans ses choix diplomatiques et politiques ne souhaitait pas ce surgissement du nationalisme albanais. Le retour à une situation mieux contrôlée exprime finalement plus précisément la politique de la France dans cette partie de l'Albanie.

\section{Les débuts d'une politique « française »}

Sous la pression de Venizélos notamment, la diplomatie reprend l'initiative et le statut de Korytza est mis en sommeil. L'armée reprend peu à peu le contrôle complet de 
l'administration et le protocole du 10 décembre 1916 est finalement aboli le 18 février 1918. Thémistocle Germenji, dont nous avons vu le rôle et les ambitions est envoyé à Salonique, jugé par un tribunal militaire et exécuté. La sévérité de cette décision semble indiquer que la France a choisi d'écouter ses alliés venizélistes, entrés en guerre au côté des Alliés le 12 juin 1917, et qui appréciaient peu l'homme fort de Korytza. Néanmoins, tout laisse penser que, sur place, les Français jouent leur propre jeu.

Peu à peu, les militaires français s'installent en effet dans la durée. Dès novembre 1917, le Général Salle, qui commande le territoire, parle de « favoriser » le développement de la langue française, du prestige de la France qui établit dans cette zone le calme et la sécurité. Les instructions reçues sont celles d'une «stricte neutralité, mais l'influence française doit prédominer sur tous les territoires qui nous sont confiés $»^{18}$. On mesure toute l'ambiguïté de ces instructions. Le "Conseil des notables" qui remplace le Conseil d'Administration mis en place par Descoins n'est plus un partenaire investi de pouvoirs importants, mais un « auxiliaire précieux » de l'administration militaire française. Son rôle est en effet clairement délimité : «son rôle politique a été nul. Nous ne l'eussions pas toléré $»^{19}$. En même temps qu'elle prétend faire taire la politique et ne viser que des objectifs militaires, la France établit sa propre politique. Elle pense s'assurer le soutien des populations locales en favorisant l'ordre, la sécurité, en permettant à chacun de satisfaire ses besoins alimentaires. C'est Franchet d'Esperey ${ }^{20}$ lui-même qui écrit au ministre de la Guerre pour que les territoires administrés par l'armée française soient bien approvisionnés en blé et ce pour des «motifs politiques ${ }^{21}$. L'administration française se rapproche davantage d'une administration coloniale. Certains de ces militaires ont d'ailleurs fait leurs premières armes dans les colonies. La plupart des troupes qui stationnent à Korytza sont issues des colonies françaises. Il ne s'agit pas, pour l'administration militaire, de donner des idées à ces " braves petits Annamites " ${ }^{22}$, aux Sénégalais et aux tirailleurs Algériens qui opèrent en Albanie.

L'exercice devient cependant de plus en plus difficile et important, les Alliés progressant sur le front d'Orient. Les territoires contrôlés par les Français s'étendent. Déjà, le premier mars 1918 était créé le territoire de Pogradec, formant avec le territoire de Korytza et la zone neutre gréco-albanaise les Confins Albanais. Enfin en septembre 1918, l'offensive victorieuse est lancée à travers les montagnes de Macédoine (Moglena, Dobropolje et Koziak). Rapidement, toute l'Albanie est libérée. Le lieutenant-colonel de Fourtou, qui commandait le territoire de Korytza, hérite d'ailleurs le 4 octobre du commandement allié de la ville de Shkodër. Les enjeux changent, mais la France reste présente, notamment à Korytza.

La fin de la guerre annonce le retour des différentes propagandes à Korytza, dans l'attente des décisions du Congrès de la Paix. L'incertitude de la politique à venir amène les Français à se placer en protecteurs. Une fois encore, les militaires pensent pouvoir faire taire la politique et être les mieux placés pour le faire. Dans son rapport du mois de décembre 1918, le chef d'escadrons Reynard-Lespinasse, commandant les Confins Albanais, décrit cette prétention : "dans ces conditions, je crois qu'une attitude nettement française, se tenant au-dessus des partis, mais ne les ignorant pas, peut seule donner une autorité morale suffisante pour maintenir le pays dans le calme jusqu'au traité de paix et pour faire accepter, avec le minimum de troubles, les décisions du congrès, quelles qu'elles soient $\aleph^{23}$.

On mesure ici toute l'ambiguïté de la position française qui se veut en même temps d'une neutralité parfaite, mais qui semble vouloir avancer ses pions et faire progresser 
son influence sur les plans économique, mais aussi intellectuel. Le commandant des Confins parle d'une "attitude nettement française", difficilement définissable, s'apparentant à « une certaine idée de la France ».

Le même, dans son rapport mensuel de juin 1919, dresse une sorte de bilan de la présence des militaires français à Korytza depuis l'automne 1916 et le prolonge en évoquant des perspectives qui sont loin d'être désintéressées. Les objectifs militaires de l'occupation du territoire semblent loin à la lecture de ces lignes :

L'influence française est nettement assise dans le pays. Ce résultat est dû :

1) à l'honnêteté de notre administration,

2) à notre respect de la liberté de conscience,

3) à l'essor donné à l'instruction publique,

4) à la sécurité que nous avons établie dans le pays et à la prospérité économique qui en est découlée.

La langue française est étudiée dans toutes les écoles albanaises. Enfin le Français inspire confiance et sympathie à la population du pays.

Cette influence a même fait tâche d'huile et dépassé les limites de notre territoire. L'intérêt de la situation acquise a été jusqu'à maintenant d'obliger les Serbes et les Grecs à respecter les limites fixées à Londres et de ce fait, d'éviter des conflits armés entre eux et les Albanais; de diminuer en formant tampon les points de frottements entre Italiens, Serbes et Grecs.

Pour l'avenir, l'influence acquise peut nous assurer un débouché pour de nombreuses branches de notre industrie, et le développement de l'étude de la langue française.

De nombreux Albanais, qui jusqu'à ces derniers temps, allaient apporter aux Etats-Unis leur main-d'œuvre et leur activité commerciale, se rendront en France nous apporter l'appoint précieux de leurs bras. ${ }^{24}$

Après une période où a dominé une stratégie plus simplement militaire, le lyrisme semble de retour, avec des relents de colonialisme. La France, à travers ses militaires, se veut pacificatrice car se situant au-dessus des partis et des propagandes. Il est désormais clair que la France poursuit une politique d'influence en Albanie. Les militaires ont pris goût à l'administration et ne souhaitent pas que cette "influence" reste sans lendemain. Franchet d'Esperey décrit en décembre 1918 ce qu'il pense être l'état d'esprit du parti nationaliste qui est selon lui minoritaire à Korytza: "les Albanais nationalistes se préoccupent du régime qui sera donné à leur pays et redoutent le départ des Français. Ils manifestent leur désir de voir Korytza "capitale naturelle de l'Albanie du sud" ne pas être disjointe, à la Paix générale, de l'Albanie reconstituée $»^{25}$.

Voici donc la situation à Korytza. Mais la guerre finie, le sort de l'Albanie en général n'est pas réglé. De nouveau, la détermination de la politique française face à la question albanaise revient en haut lieu. L'action et la réflexion françaises se détachent de nouveau de l'observation sur le terrain pour se rapprocher des enjeux internationaux. Certes, des hommes comme Krajewski tentent de tirer la diplomatie française vers davantage de reconnaissance des droits des Albanais (le représentant de la France auprès d'Essad fait en l'occurrence la part belle à cet homme de plus en plus discrédité en Albanie même). C'est l'objet de son rapport remis au ministre Pichon sur «la question albanaise devant le congrès de la paix ${ }^{26}$, rédigé à Paris en tant que délégué à 
la Commission de contrôle en Albanie. Mais c'est désormais à Paris que l'on va décider du sort de l'Albanie. du Congrès de la Paix qui s'ouvre à Paris le 18 janvier 1919.

\section{ANNEXES}

\section{[Lettre du Colonel Descoins au Général Leblois, commandant l'Armée Française d'Orient, à Monastir] ${ }^{27}$}

Korytza, 10 décembre 1916

$\mathrm{N}^{\circ} 178 / \mathrm{S}$

J'ai l'honneur de vous rendre compte de ce qui suit :

Ainsi que je le prévoyais, la question Albanaise s'est posée à Korytza sous la forme définie au paragraphe 2 de mon rapport $n^{\circ} 132 / \mathrm{s}$ du 6 décembre 1916.

J'ai reçu ce matin, 10 décembre, la visite de 14 délégués de la population L'un d'eux s'est exprimé en ces termes :

« Nous,

délégués du Peuple Albanais, venons déclarer au représentant de la France que le Kaza de Korytza avec ses dépendances de Biklista, Kolonia, Opari et Gora, est désormais une province autonome administrée par des fonctionnaires Albanais. Nous demandons à nous placer sous la protection de l'autorité militaire Française. »

La déclaration et la demande n'ayant rien de contraire à la note $\mathrm{N}^{\circ} 2995$ (C.A.A.) du 29 novembre 1916 portant constitution du Territoire militaire à Korytza, j'ai répondu :

«Vous venez m'exprimer la volonté du peuple Albanais du Kaza de Korytza de vivre désormais libre et vous demandez la protection de l'Armée Française. Comme représentant du Commandement Français, j'acquiesce à votre demande. Je déclare donc qu'à dater d'aujourd'hui 10 décembre, le Kaza de Korytza, avec ses dépendances de Biklista, Kolonia, Opari et Gora, est constitué en province autonome, administrée par des fonctionnaires Albanais, sous la protection de l'autorité militaire Française. »

En conséquence, les délégués et moi avons rédigé et signé le protocole dont une expédition est ci-jointe.

L'administration et la police du territoire sont, dès à présent, assurées dans les conditions indiquées au protocole.

L'ordre et le calme les plus complets n'ont pas cessé de régner en ville.

Salih Budka, mis au courant des événements m’a fait aviser qu'il se rangeait à nos côtés. 


\section{Protocole}

Korytza, 10 décembre 1916

I. Conformément au voeu du peuple Albanais exprimé par ses délégués, le Kaza de Korytza, avec ses dépendances de Biklista, Kolonia, Opari et Gora, est constitué en province autonome, administrée par des fonctionnaires Albanais, sous la protection de l'autorité militaire Française.

II. L'administration du Kaza est confiée à un conseil de 14 membres, mi-partie chrétiens, mi-partie musulmans. Ce Conseil est chargé :

1) de l'étude de toutes les mesures ayant en vue la bonne administration du Kaza de Korytza et de ses dépendances;

2) du contrôle du fonctionnement de tous les services publics.

III. Le Conseil proposera à l'acceptation de l'autorité militaire Française les noms des titulaires des différents emplois. Les nominations à ces emplois seront faites par l'autorité militaire Française.

IV. Il sera institué, sous l'autorité d'un Préfet de police, un corps de gendarmerie-police chargé de maintenir l'ordre à l'intérieur.

V. Il sera créé un corps de " gendarmerie mobile albanaise » chargé de sauvegarder l'indépendance du territoire et la liberté de ses habitants.

VI. Il pourra être également créé, dans le même but, des corps réguliers de volontaires dont l'effectif dépendra des circonstances et des crédits disponibles.

VII. Lorsqu'elles seront appelées à agir, les forces de police, la gendarmerie mobile et les troupes de volontaires seront sous l'autorité supérieure de l'officier français commandant le Cercle de Korytza.

VIII. La langue officielle sera l'albanais.

IX. Le drapeau du Kaza de Korytza sera l'étendard traditionnel de Scanderbeg avec cravate tricolore aux couleurs françaises.

Pour la population, les délégués : [Noms des délégués]

Pour l'autorité militaire Française : Colonel Descoins, commandant militaire de Korytza.

\section{NOTES}

1. Nous disposons pour tous ces faits du témoignage qu'il a lui même donné en 1929 : Descoins (Général), «Six mois d'histoire de l'Albanie », Revue d'histoire de la guerre mondiale, 7 (4), octobre 1929, et 8 (1), janvier 1930.

2. Rapport sur la situation de Korytza du 23 novembre 1916 in ibid., p. 325.

3. Ministère des Affaires Etrangères (M.A.E.), Série A-Guerre 14-18 Italie 561 (avril 1915). Il faut d'ailleurs constater que c'est le nom grec de la ville qui est utilisé par les Français, de même que les villes côtières (Vlorë, Durrës, Shkodër) sont toujours appelées de leur nom italien (Vallona, Durazzo, Scutari). 
4. Il a été commandant avant la guerre d'une brigade de cavalerie hellénique

5. Descoins (Général), art. cit., p. 332.

6. Ibid.

7. Service historique de l'armée de terre (S.H.A.T.) $20 \mathrm{~N} 845$ Unités diverses.

8. Télégramme envoyé le 15 décembre par Sarrail au M.A.E., cité dans Sarrail, Mon commandement en Orient, Paris : Flammarion, 1920, p. 222.

9. Le texte du protocole et la lettre par laquelle Descoins en informe ses supérieurs se trouvent en annexe

10. Descoins (Général), art. cit., p. 339.

11. S.H.A.T., 5 N 197 Cabinet du Ministre, « Descoins au général commandant l'A.F.O.».

12. Des timbres portant la mention « Republika Shqipetare » sont imprimés de mai à novembre 1917 (S.H.A.T. 20 N 850 Unités diverses).

13. Descoins (Général), art. cit., p. 36.

14. S.H.A.T. 20 N 850-8 Unités diverses, «Rapport de novembre 1917 sur l'administration du Caza de Korytza ».

15. Carcopino (Jérôme), Souvenirs de la guerre en Orient. 1915-1917, Paris : Hachette, 1970.

16. M.A.E. Europe 1918-1929 Albanie 7 Politique intérieure, 10/06/20.

17. S.H.A.T. 20 N 843 Unités diverses, Ordres généraux, 03/06/18.

18. S.H.A.T. $20 \mathrm{~N} 849$ Unités diverses, Correspondance, 18/11/18, "Commandant du territoire de Korytza au général Commandant l'A.F.O.».

19. S.H.AT. $20 \mathrm{~N} 850$ Unités diverses, «Rapport sur l'administration du territoire de Korytza pendant le mois d'avril $1918 »$.

20. Il est le Commandant des Armées Alliées depuis le 18 juin 1918, en remplacement du Général Guillaumat

21. S.H.A.T. 5 N 197 Cabinet du Ministre, «Télégramme ( $n^{\circ}$ 767) du Général Franchet d'Esperey (C.A.A.) au ministre de la Guerre daté du 8 novembre 1918 ».

22. C'est l'expression employée par le colonel Descoins (Descoins (Général), art. cit., p. 34).

23. S.H.AT. $20 \mathrm{~N} 849$ Unités diverses, «Rapport sur l'administration du territoire de Korytza pendant le mois de décembre 1918, 4 janvier $1919 »$, p. 2.

24. S.H.A.T. $20 N 849$ Unités diverses, «Rapport sur l'administration du territoire de Korytza pendant le mois de juin 1919, 6 juillet $1919 »$.

25. Les armées alliées en Orient après l'armistice de 1918 (Comptes-rendus mensuels adressés par le général C.A.A. à l'état-major de l'armée à Paris de décembre 1918 à octobre 1920), réunis par le général J. Bernachot, S.H.A.T. 1972, p. 99.

26. M.A.E. série A-Paix 187 Conditions de la paix, « Albanie », 31/12/18.

27. S.H.A.T. 5 N 197 Cabinet du Ministre.

INDEX

Keywords : Albanie, Première Guerre mondiale Index géographique: Albanie 